\title{
The phylogenetic relationships of Caenorhabditis and other rhabditids*
}

\author{
Karin Kiontke ${ }^{\S}$, David H. A. Fitch ${ }^{\S}$, Department of Biology, New York \\ University, New York, NY 10003 USA
}

\section{Table of Contents}

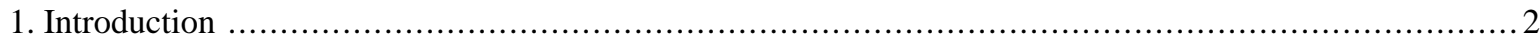

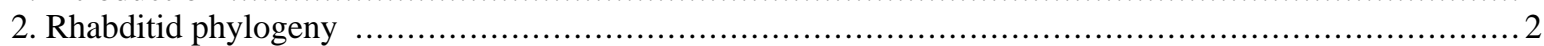

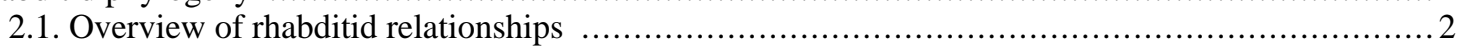

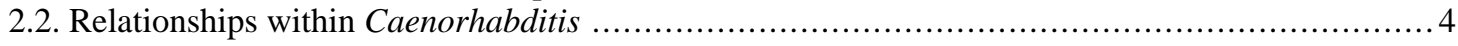

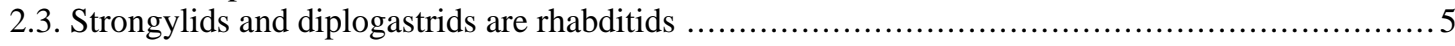

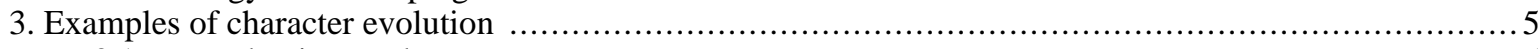

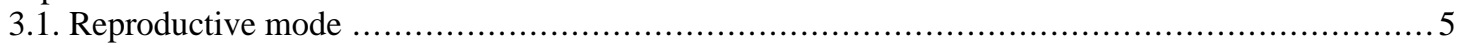

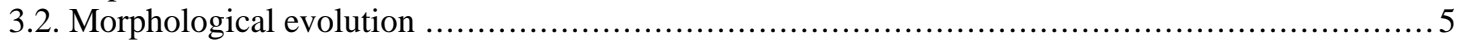

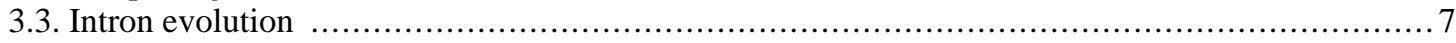

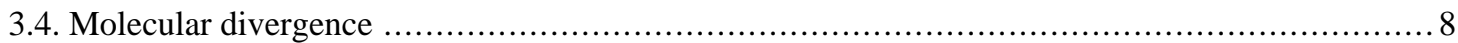

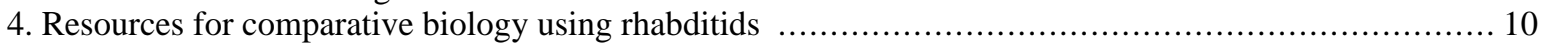

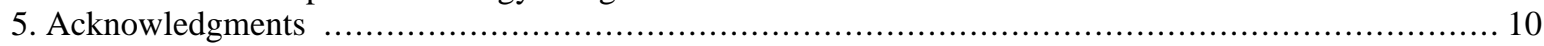

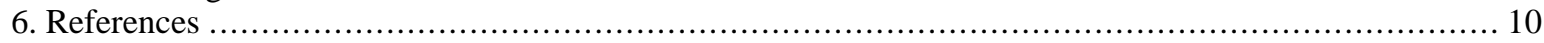

\begin{abstract}
C. elegans is a member of a group of nematodes called rhabditids, which encompasses a large number of ecologically and genetically diverse species. A new, preliminary phylogenetic analysis is presented for concatenated sequences of three nuclear genes for 48 rhabditid and diplogastrid species (including 10 Caenorhabditis species), as well as four species representing the outgroup. Although many relationships are well-resolved, more data are still needed to resolve some key relationships, particularly near the base of the rhabditid tree. There is high confidence for two major clades: (1) a clade comprising Mesorhabditis Parasitorhabditis, Pelodera, Teratorhabditis plus a few other species; (2) a large clade (Eurhabditis) comprising most of the remaining rhabditid genera, including Caenorhabditis and its sistergroup Protorhabditis-Prodontorhabditis-Diploscapter. Eurhabditis also contains the parasitic strongylids, the entomopathogenic Heterorhabditis, and the monophyletic group Oscheius which includes the satellite model organism $O$. tipulae. The relationships within Caenorhabditis are well resolved. The analysis also suggests that
\end{abstract}

\footnotetext{
*Edited by Lisa R. Girard. Last revised July 27, 2005. Published August 11, 2005. This chapter should be cited as: Kiontke, K. and Fitch, D.H.A. The Phylogenetic relationships of Caenorhabditis and other rhabditids (August 11, 2005), WormBook, ed. The C. elegans Research Community, WormBook, doi/10.1895/wormbook.1.11.1, http://www.wormbook.org.

Copyright: (C) 2005 Karin Kiontke and David H. A. Fitch. This is an open-access article distributed under the terms of the Creative Commons Attribution License, which permits unrestricted use, distribution, and reproduction in any medium, provided the original author and source are credited.

${ }^{\S}$ To whom correspondence should be addressed. E-mail: kk52@nyu.edu or david.fitch@ nyu.edu
} 
rhabditids include diplogastrids, to which the second satellite model organism Pristionchus pacificus belongs. Genetic disparity within Caenorhabditis is as great as that across vertebrates, suggesting Caenorhabditis lineages are quickly evolving, ancient, or both. The phylogenetic tree can be used to reconstruct evolutionary events within rhabditids. For instance, the reproductive mode changed multiple times from gonochorism to hermaphroditism, but only once from hermaphroditism to gonochorism. Complete retraction of the male tail tip, leading to a blunt, peloderan tail, evolved at least once. Reversions to unretracted tail tips occurred within both major rhabditid groups. The phylogeny also provides a guide to species which would be good candidates for future genome projects and comparative studies.

\section{Introduction ${ }^{\dagger}$}

Caenorhabditis belongs to a large group of primarily bacteriophagous nematodes called rhabditids (Figure 1). This taxon comprises a great diversity of species, including some parasites (see A quick tour of nematode diversity and the backbone of nematode phylogeny). Resolving rhabditid phylogeny is key to uncovering how evolutionary changes occurred in this diversification. A phylogeny for rhabditids also provides an important foundation for comparative biology using C. elegans, "satellite" model systems Pristionchus pacificus, Oscheius tipulae, and other Caenorhabditis species with sequenced genomes (C. briggsae, C. remanei, $C$. japonica, and Caenorhabditis n. sp. represented by strain CB5161 among others; see Evolution of development in nematodes related to C. elegans). The selection of appropriate representative taxa for further genome sequencing projects also depends on an accurate phylogeny.

Phylogenetic analyses using DNA sequences of small subunit ribosomal RNA (SSU rRNA) genes have revealed some relationships that are inconsistent, as well as some that are consistent, with earlier phylogenies (Fitch et al., 1995; Blaxter et al., 1998; Sudhaus and Fitch, 2001). Many relationships, however, have not been sufficiently resolved. It is therefore important to expand the data pool with regard to both loci and taxa.

Here we report conclusions of an analysis (which will be published elsewhere) using three genes for rhabditids and their closest relatives. In this phylogeny, several branches are well supported. For example, most species belong to either of two major monophyletic groups, here called "Eurhabditis" and "Pleiorhabditis", both of which show high support in this analysis. Eurhabditis includes strongylids and Heterorhabditis, as well as Caenorhabditis and its sister taxon, the Protorhabditis group. There is also some evidence that diplogastrids (e.g., Pristionchus) are part of rhabditids. As we accumulate more data, we are gaining a clearer picture of rhabditid phylogeny. There is still much that remains mysterious, however. In particular, it will be important to establish who are the closest relatives of rhabditids, to sort out the earliest lineages of rhabditids, and to further elucidate the relationships among members of the Eurhabditis group. Only after this is achieved should the much-needed taxonomic revision be initiated. At the moment, however, it is still too early to attempt such a classification because a number of relationships are not sufficiently resolved.

A well-resolved phylogeny is an essential step in inferring the evolutionary changes that have occurred and for discovering possible correlations between such changes. We provide examples of how the phylogeny so far has provided insight into the evolution of reproductive mode, morphological evolution in the male tail tip, the evolution of introns, and molecular divergence. Database and strain resources for rhabditids are being developed to support comparative work with rhabditids.

\section{Rhabditid phylogeny}

\subsection{Overview of rhabditid relationships}

A preliminary phylogenetic hypothesis for representative rhabditid species and four species from the outgroup is shown in Figure 1. The analysis is based on DNA sequences from nearly complete small and large subunit (SSU and LSU) ribosomal RNA (rRNA) genes and a portion of the RNA Pol II (RNAP2) gene (KK and DF, unpublished; original data and details of the analysis will be published elsewhere).

\footnotetext{
${ }^{\dagger}$ Terms used: taxon (plural taxa)- a group of phylogenetically related species; monophyletic- a group of species that includes all descendants of one ancestor; paraphyletic- a group of species that includes some but not all descendants of an ancestor; polyphyletic- a group of species that includes descendants of more than one ancestor; clade- a monophyletic taxon.
} 


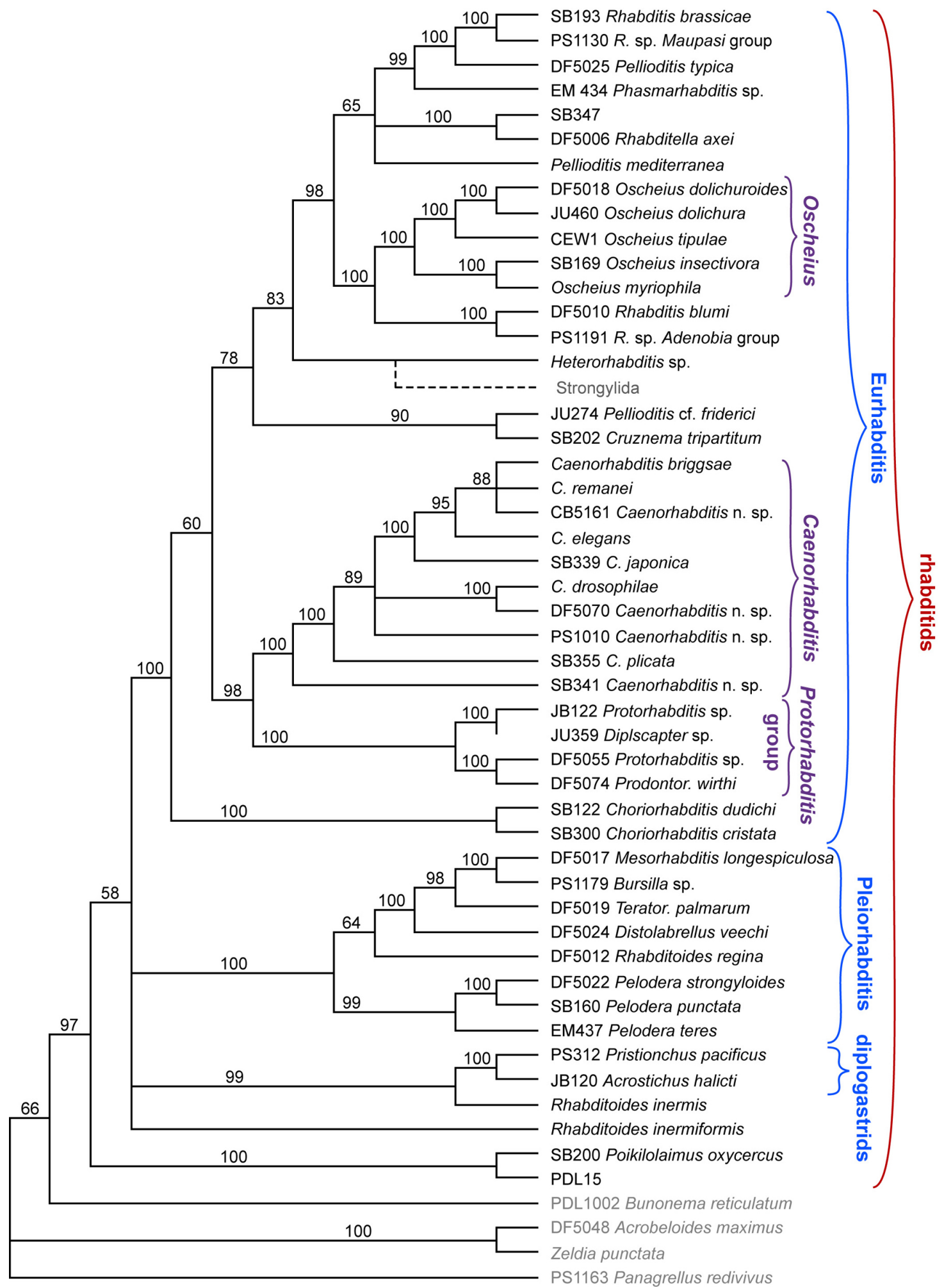

Figure 1. Rhabditid phylogeny as inferred by weighted parsimony jackknife analysis using DNA sequences from three genes (SSU and LSU rRNA and RNA polymerase II). Strain designations are given for species available as stocks. Gray taxa names designate outgroup representatives. Some of the clearer monophyletic groups are delineated with brackets. Numbers on the branches indicate the percentage of 1,000 jackknife replicates in which the branch appeared (50\% of randomly chosen sites were deleted in each replicate). For each jackknife replicate, four random sequences of taxon addition were used to generate starting trees. Branches were collapsed where jackknife values were below 50\%. The position of the strongylid clade, inferred from previous analyses (Blaxter et al., 1998; Fitch and Thomas, 1997), is shown as a dashed line. (KK and DF, unpublished; details of the analysis will be published elsewhere.). Right-click or control-click to see larger image. 
Two major clades of rhabditids can be distinguished, called here "Pleiorhabditis" and "Eurhabditis" (Figure 1). Although these two groups were combined in a single monophyletic group (supported by $87 \%$ bootstrap value) in an earlier analysis using only SSU rRNA genes (Sudhaus and Fitch, 2001), such a clade does not appear in the present jackknife analysis with additional genes. Pleiorhabditis comprises several genera of species with a posterior vulva (e.g., Mesorhabditis, Teratorhabditis) and also the genus Pelodera which includes Pelodera strongyloides, reported to be a facultative parasite of mammals (Sudhaus and Schulte, 1988; Jones et al., 1991). Eurhabditis includes almost all the other rhabditid species, including Caenorhabditis, and thus differs from the Eurhabditis originally proposed by Sudhaus on the basis of a different view of relationships (see Sudhaus and Fitch, 2001). Additionally, Eurhabditis includes the "Protorhabditis group", named after its most prominent members, which is the sister clade to Caenorhabditis. Eurhabditis includes a monophyletic genus Oscheius that comprises two separate clades, one of leptoderan species, the Insectivora group, and another of peloderan species, the Dolichura group, as proposed by Sudhaus and Hooper (1994; see also Figure 4). One member of this latter group, Oscheius tipulae (represented here by strain CEW1) is used as a satellite model organism for comparative studies of development (see Evolution of development in nematodes related to C. elegans; Sommer, 2000) and molecular biology (e.g. Evans et al., 1997). The strongylid parasites of vertebrates and the Heterorhabditis pathogens of insects have also clearly evolved from within the Eurhabditis clade (see A quick tour of nematode diversity and the backbone of nematode phylogeny).

\subsection{Relationships within Caenorhabditis}

Relationships are now well-resolved among the Caenorhabditis species which are represented by live strains (Figure 2; Kiontke et al., 2004; Cho et al., 2004). Of these species, C. briggsae and C. remanei are closest relatives. No single species is most closely related to $C$. elegans, which is the sister species of a clade that includes $C$. briggsae, $C$. remanei, and Caenorhabditis n. sp. (represented here by strain CB5161). The closest species to these four Elegans group species is $C$. japonica, which was chosen for a genome sequencing project in order to provide an outgroup comparison for the four Elegans group species. Another undescribed new species (represented here by strain PS1010), which has been used in some comparisons (e.g. Baldwin et al., 1997), diverged earlier than $C$. japonica. Uncertainty still exists for some Caenorhabditis relationships; instead of being related in the manner depicted in Figure 2, C. sp. PS1010 could be most closely related to C. drosophilae and Caenorhabditis n. sp. (DF5070) (Kiontke et al., 2004). For more about Caenorhabditis biodiversity and a phylogenetic hypothesis incorporating other Caenorhabditis species, see Ecology of Caenorhabditis species (also Sudhaus and Kiontke, 1996).

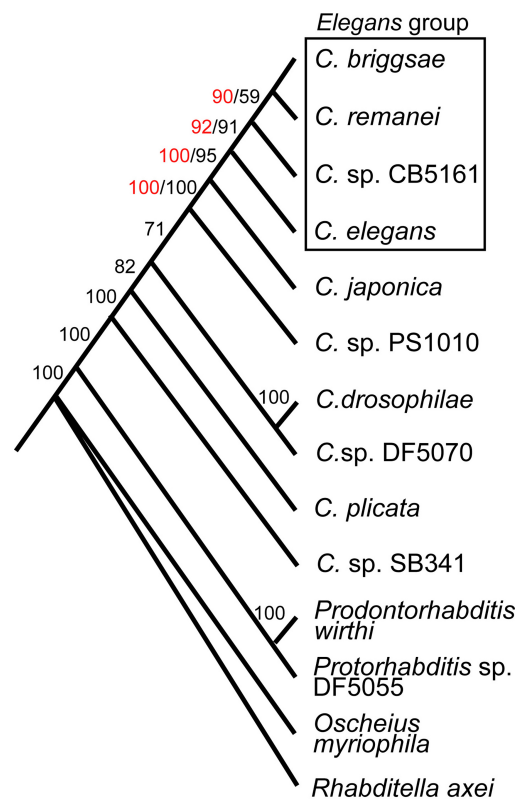

Figure 2. Phylogeny of Caenorhabditis species represented by living strains and four outgroup representatives. Black numbers on the branches denote percentage jackknife support from 2,000 replicates for an analysis incorporating all taxa shown using SSU and LSU rRNA genes and RNAP2 with the third codon positions deleted. Red numbers denote jackknife support in 5,000 replicates for an analysis that included the seven most closely related Caenorhabditis species for SSU and LSU rRNA genes, and coding regions from genes for RNAP2, par-6, and pkc-3 with the third codon positions included. Third codon positions were only saturated with substitutions in pairwise comparisons involving species less closely related to $C$. elegans than $C$. sp. PS1010. (Modified from Kiontke et al., 2004). Right-click or control-click to see larger image. 


\subsection{Strongylids and diplogastrids are rhabditids}

Rhabditids, as a monophyletic group, include two taxa that have been previously classified separately. First, the strongylids are a group of vertebrate parasites traditionally considered outside of rhabditids. Molecular analyses have placed strongylids clearly within the Eurhabditis group of rhabditids and as a sister group of the insect pathogenic Heterorhabditis (Fitch and Thomas, 1997; Blaxter et al., 1998; Sudhaus and Fitch, 2001; De Ley and Blaxter, 2002).

A second group of species traditionally classified outside of rhabditids is the group designated here informally as diplogastrids (called Diplogastrina, Diplogastridae, or Diplogasteromorpha in formal classification systems), which includes the satellite model system, Pristionchus pacificus (see Evolution of development in nematodes related to $C$. elegans). Specifically, there appears to be a relationship between diplogastrids and Rhabditoides inermis, a rhabditid (see also Sudhaus and Fitch, 2001). According to this phylogeny, R. inermis is more closely related to diplogastrids than to its traditional congener, Rhabditoides inermiformis, despite their morphological similarities. (Another species, Rhabditoides regina, resides within Pleiorhabditis, thus making "Rhabditoides" polyphyletic.) Furthermore, our phylogeny suggests that Poikilolaimus could be in the most anciently diverged lineage of rhabditids, placing diplogastrids distinctly within rhabditids, although the jackknife support (58\%) is not strong for this conclusion. Together, these data suggest that diplogastrids are not a clade separate from rhabditids, but are instead derived from within rhabditids. These data contradict a recent proposal based on morphology that bunonematids are the sister taxon of diplogastrids (Fürst von Lieven, 2002).

\section{Examples of character evolution}

\subsection{Reproductive mode}

Using the molecular phylogeny, we have traced the evolution of gonochorism (males and females must cross to propagate), self-fertile hermaphroditism (see Sex-determination in the germ line), heterogony (alternating generations of gonochorism and hermaphroditism), and parthenogenesis (sperm, if they exist, do not contribute genetic material; Figure 3). Among the taxa represented in Figure 3, we find that gonochorism is ancestral and that hermaphroditism has arisen at least ten times independently in all the major rhabditid clades, with only one possible reversal back to gonochorism occurring within the Dolichura species group of Oscheius. Such massive convergence provides an excellent opportunity to study how significantly developmental constraints limit or channel evolution.

According to the most parsimonious reconstruction, and assuming reversibility and equal chances of changes between character states, C. briggsae and C. elegans evolved hermaphroditism independently (Kiontke et al., 2004). If we consider that changes from gonochorism to hermaphroditism have occurred ten times more frequently than the reverse in rhabditids, it becomes even more plausible that hermaphroditism evolved independently in these two species. In such a case, there are two possibilities for evolutionary change: parallelism (in which independent changes affect the same mechanism) or convergence (in which the changes affect different mechanisms but yield similar results). In the case of $C$. elegans and $C$. briggsae, hermaphroditism appears to be convergent because there is a fundamental difference between the two species in the identity of FOG-2, for which no single ortholog exists in C. briggsae, and developmental role of GLD-1, which inhibits female fate in the germline of $C$. elegans but promotes female fate in C. briggsae (Nayak et al., 2005; see Sex-determination in the germ line).

We also find that heterogony evolved twice (in Heterorhabditis and an undescribed species represented by strain SB347) and parthenogenesis evolved once (within the Protorhabditis group).

\subsection{Morphological evolution}

As an example of morphological evolution, we have traced the history of changes in morphogenesis of the male tail tip. In $C$. elegans, the shapes of the hypodermal cells in the male tail tip change dramatically as the cells fuse during L4 male morphogenesis and the conically pointed tail tip of the larva retracts to form the rounded, "peloderan" tail of the adult male (Nguyen et al., 1999). Tail morphogenesis does not occur in hermaphrodites or in females of most other rhabditid species. Although males of most rhabditid species do undergo tail morphogenesis, the tip of the tail does not always retract. In such cases, the tail tip remains pointed, as in larvae, usually sticking out beyond the posterior edge of the fan or bursa, if a fan exists. This tail form is called "leptoderan". 


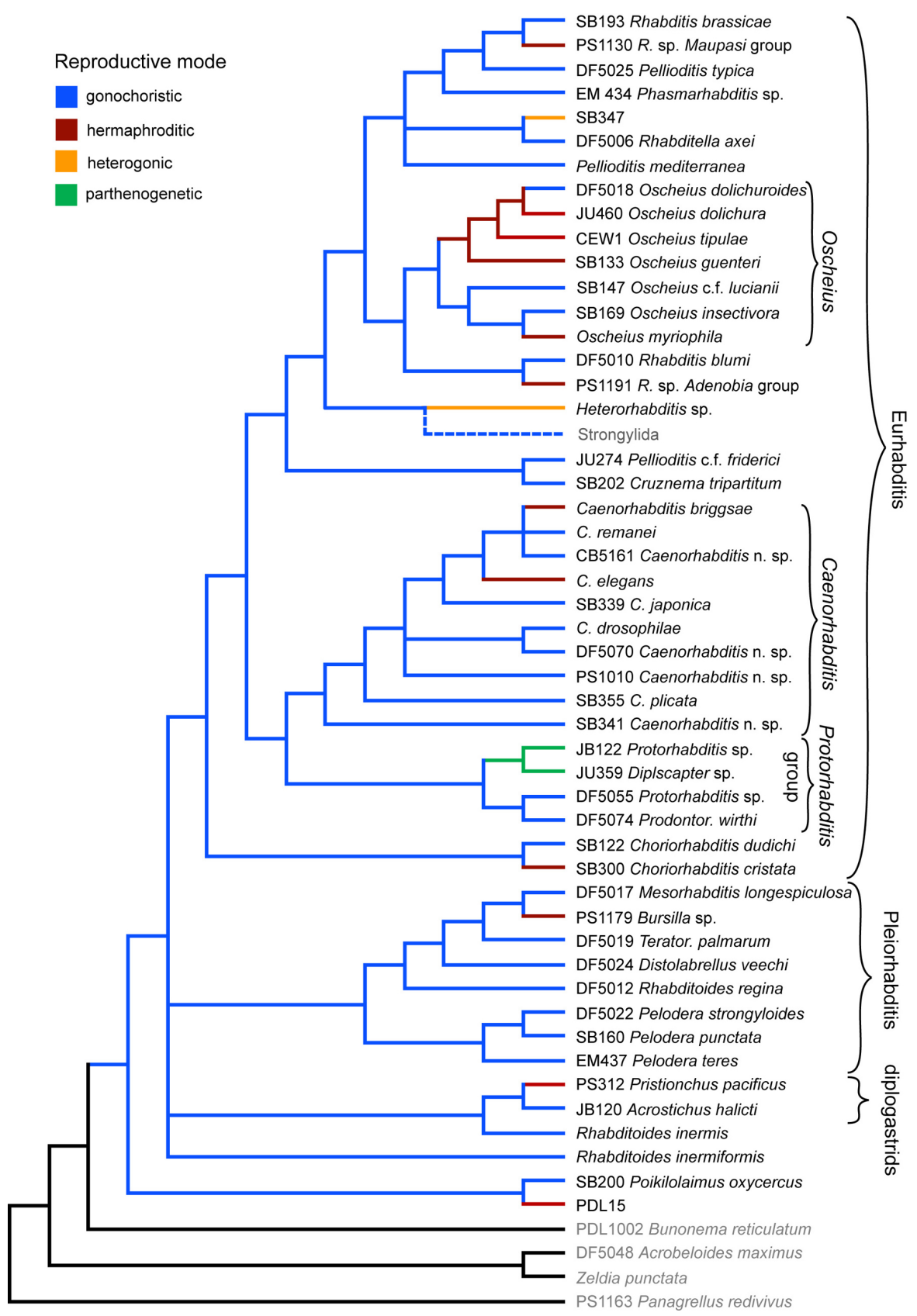

Figure 3. Evolution of reproductive mode. Reproductive modes were determined for the terminal taxa and mapped onto the molecular phylogeny depicted in Figure 1. Two Oscheius species and the lineage to strongylids (dashed lines) were added according to their position in phylogenies obtained with SSU rDNA alone. Red branches represent lineages in which hermaphroditism evolved; blue branches represent lineages in which gonochorism occurred; orange indicates heterogonic mode (different generations switch between gonochoristism and hermaphroditism); green indicates probable or known parthenogens. Character states for ancestral lineages were inferred by parsimony under the assumption that character state changes are equally likely and reversible. Right-click or control-click to see larger image.

There have been at least seven independent changes in the shape of the male tail tip during rhabditid evolution (Figure 4). Because some branches are still unresolved, the directionality of most of these changes is unclear. However, the present phylogeny allows us to conclude that at least two changes occurred from peloderan to leptoderan. Some of the changes in male tail tips must be convergent because we know that peloderan tail tips can be constructed in different ways (e.g., with or without fusions of the hypodermal cells), as can leptoderan tail tips (Nguyen et al., 1999; Fitch, 1997; Fitch, 2000). Because of these convergences, it is clear that there is no monophyletic clade of leptoderan species or of peloderan species (cf. Peloderinae of Andrássy, 1983). We have found that several other morphological characters (such as lack of glottoid apparatus) show similar homoplasy at 
gross morphological levels (e.g., Sudhaus and Fitch, 2001). Thus, rhabditids provide fertile ground for investigating the role of developmental plasticity in evolution.

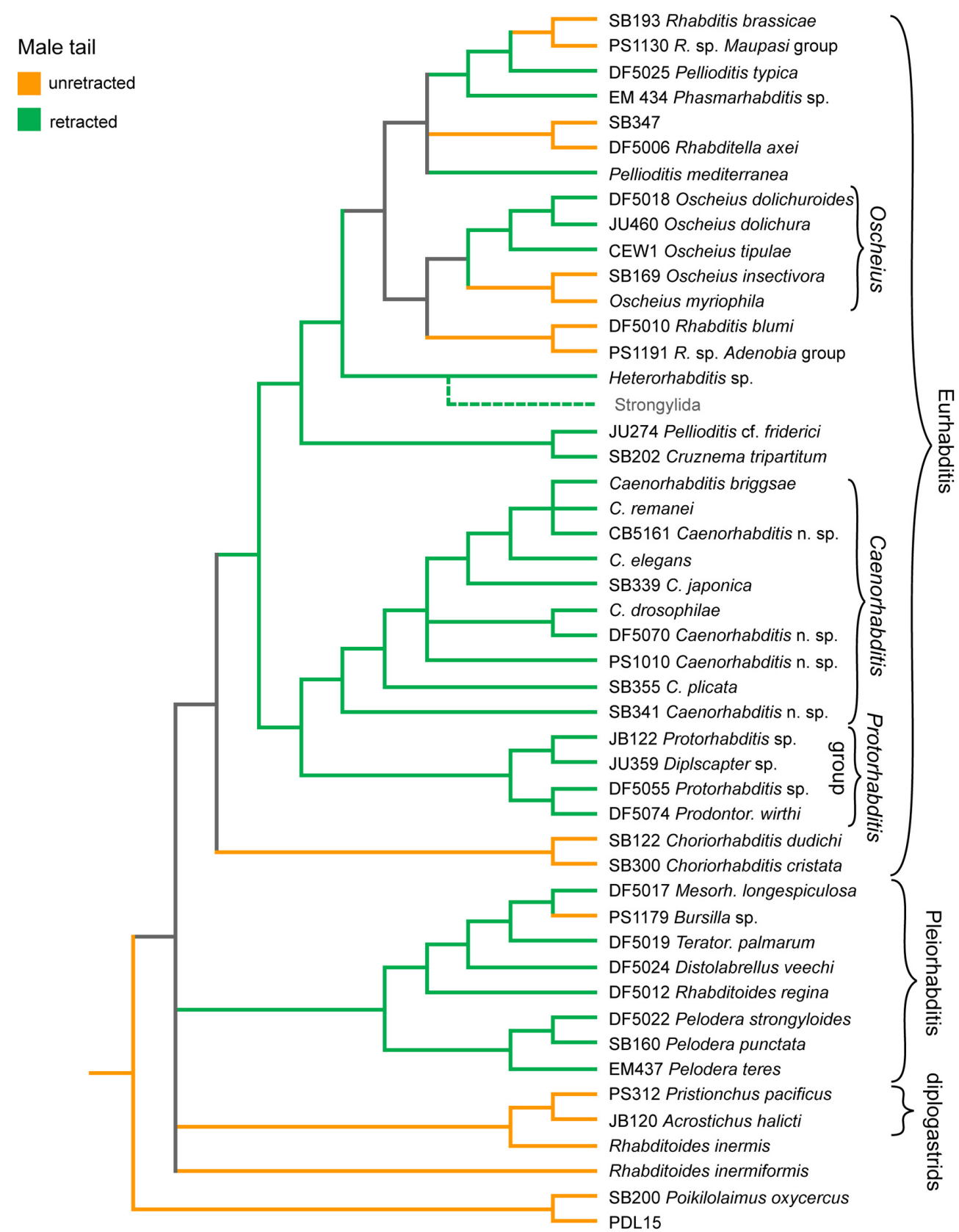

Figure 4. Evolution of male tail tip morphogenesis. Orange branches represent evolutionary lineages in which males had unretracted tail tips, which are therefore pointed in shape and referred to as "leptoderan" if there is a fan; green represents lineages in which males had retracted tail tips, which are thus rounded in shape and are referred to as "peloderan," as in C. elegans. Gray represents lineages for which the character state cannot be decided. Using the phylogeny depicted in Figure 1, ancestral states were inferred by parsimony under the assumption that character state changes are equally likely and reversible. Right-click or control-click to see larger image.

\subsection{Intron evolution}

Using the well-resolved Caenorhabditis phylogeny, we inferred the most parsimonious history of intron gains and losses in a 1,860-bp portion of the gene coding for the largest subunit of RNAP2 (Kiontke et al., 2004). This phylogeny of closely related species allows finer resolution of such events than do comparisons among distantly related model organisms, which have been the main staple of intron evolution fare (e.g. Rogozin et al., 2003). We find that intron evolution is extraordinarily dynamic (Figure 5). In this one region, the number of introns present in a 
species can range between 1 and 14. Considering only homologous intron positions at which a single most parsimonious reconstruction could be made ("unequivocal" positions) and assuming that intron gains and losses are equally likely, introns were lost 12 times and gained 4 times (Kiontke et al., 2004). If one considers the possibility that losses are more likely than gains and that it might be highly improbable for an intron to be regained in exactly the same spot, it is possible that as many as 27 losses and at most 3 gains (but at least 1 gain) occurred. Most of these intron losses occurred in the stem species of the Caenorhabditis clade, and several losses occurred later as the clade evolved (Figure 5). One intron in C. sp. CB5161 is unique among all rhabditids we have characterized so far, and is thus a clear gain (Figure 5). Characterization of the homologous region in other rhabditids suggests that intron evolution is very dynamic with periods of losses and gains (KK and DF, unpub.). Frequent intron loss in other Caenorhabditis genes has also been reported (Cho et al., 2004). Recent intron gains have also been documented, some of which have sequence similarity to other introns and could therefore have originated by reverse splicing (Coghlan and Wolfe, 2004).

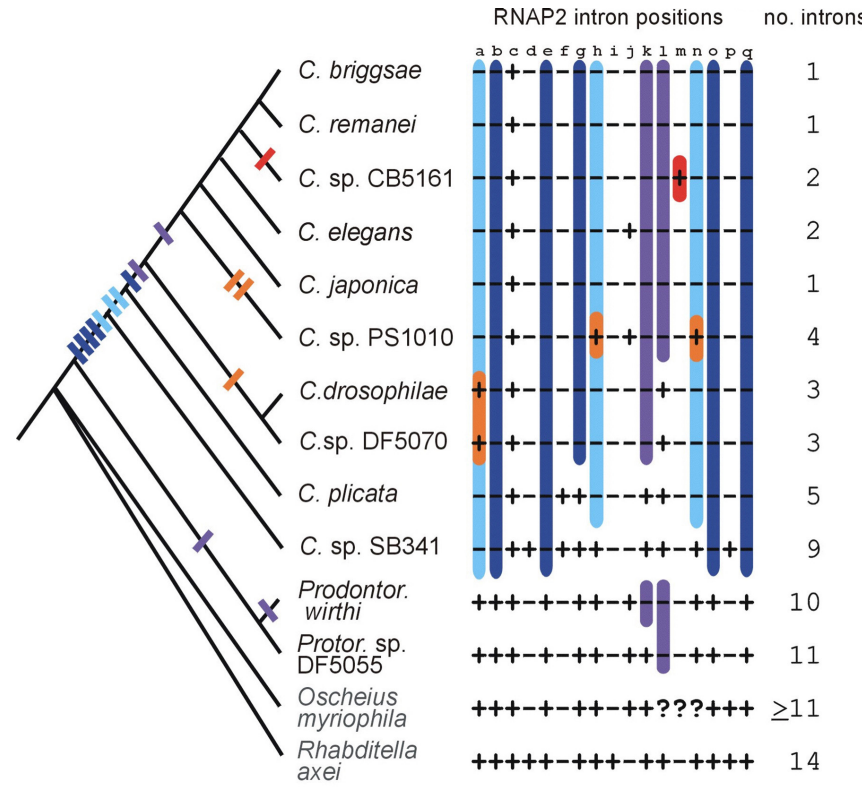

Figure 5. Intron evolution in part of the RNAP2 gene of Caenorhabditis species. The presence (+) or absence (-) of introns at particular sites (a-q) in the RNAP2 gene is depicted as a matrix (question marks indicate missing data). For sites a, b, e, g, h, k-o, and q, a single, unequivocal, most parsimonious history of intron insertion/deletion could be inferred (rectangles on the branches of the phylogeny). For the other sites, more than one most parsimonious scenario exists under the assumption that gains and losses are equally probable. Sites at which a single deletion occurred during Caenorhabditis cladogenesis are marked in dark blue; purple indicates sites at which two deletions occurred; light blue and orange indicate sites at which deletions and subsequent reinsertions could have occurred; red indicates a unique intron insertion. (Modified from Kiontke et al., 2004). Right-click or control-click to see larger image.

\subsection{Molecular divergence}

Genome-level comparisons of molecular divergence show that $C$. elegans and $C$. briggsae are a little bit less similar at the molecular level than are human and mouse (Stein et al., 2003). We find the same relative differences when comparing these species with RNAP2 and rRNA genes alone (Kiontke et al., 2004). Because many taxa are represented by SSU rRNA sequences, we can use this molecule to compare the degree of molecular divergences among other vertebrate taxa with the divergences among rhabditids. The divergence between the least closely related Caenorhabditis species ( $C$. briggsae versus $C$. sp. SB341) is comparable to the divergence between mouse and zebrafish, encompassing a broad range of vertebrate lineages (Figure 6). Furthermore, species within the Eurhabditis clade are at least as different at the molecular level as mouse and sea urchin, encompassing a broad range of deuterostome lineages. It is important to note that the Eurhabditis clade is but a small fraction of Nematoda. Thus, the nematodes diverged from other animals very anciently, rates of molecular evolution are much faster in nematodes than in deuterostomes, or both. Because there is no accurate method for calibrating a "molecular clock" for nematodes, estimates for dates of divergence will be extremely unreliable. Complicating this issue, there is considerable rate heterogeneity among different lineages for some genes (e.g., SSU rRNA; see Kiontke et al., 2004). The best measure of taxonomic difference in rhabditids is therefore simply the relative amount of sequence difference (as in Figure 6), not some date of divergence which could be very inaccurate. 


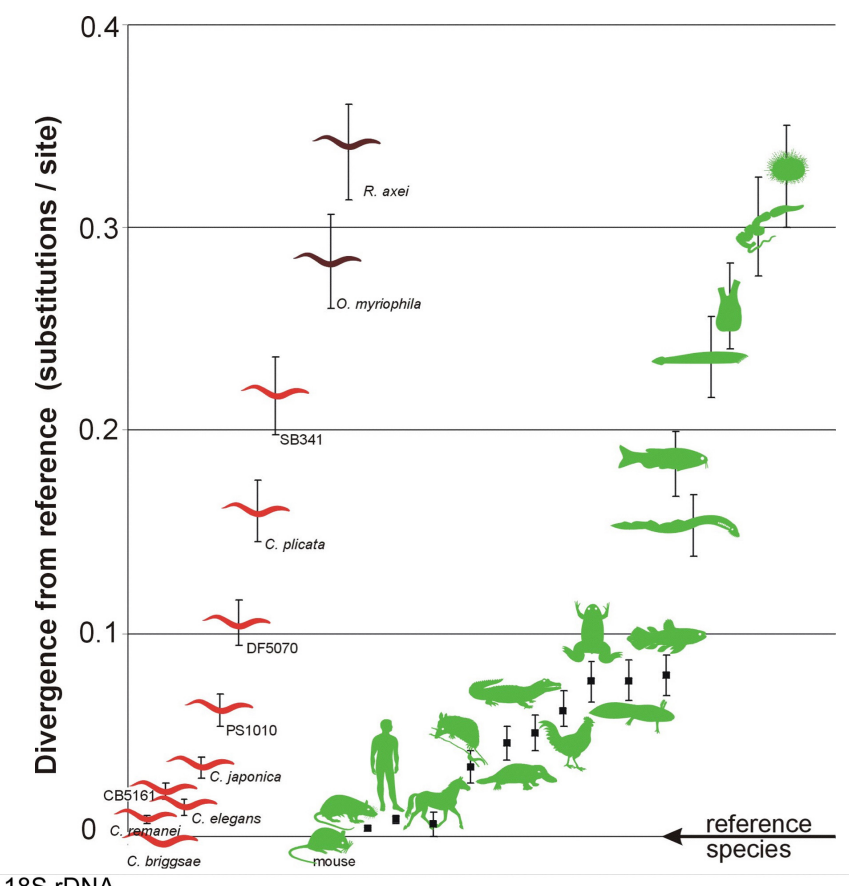

18S rDNA
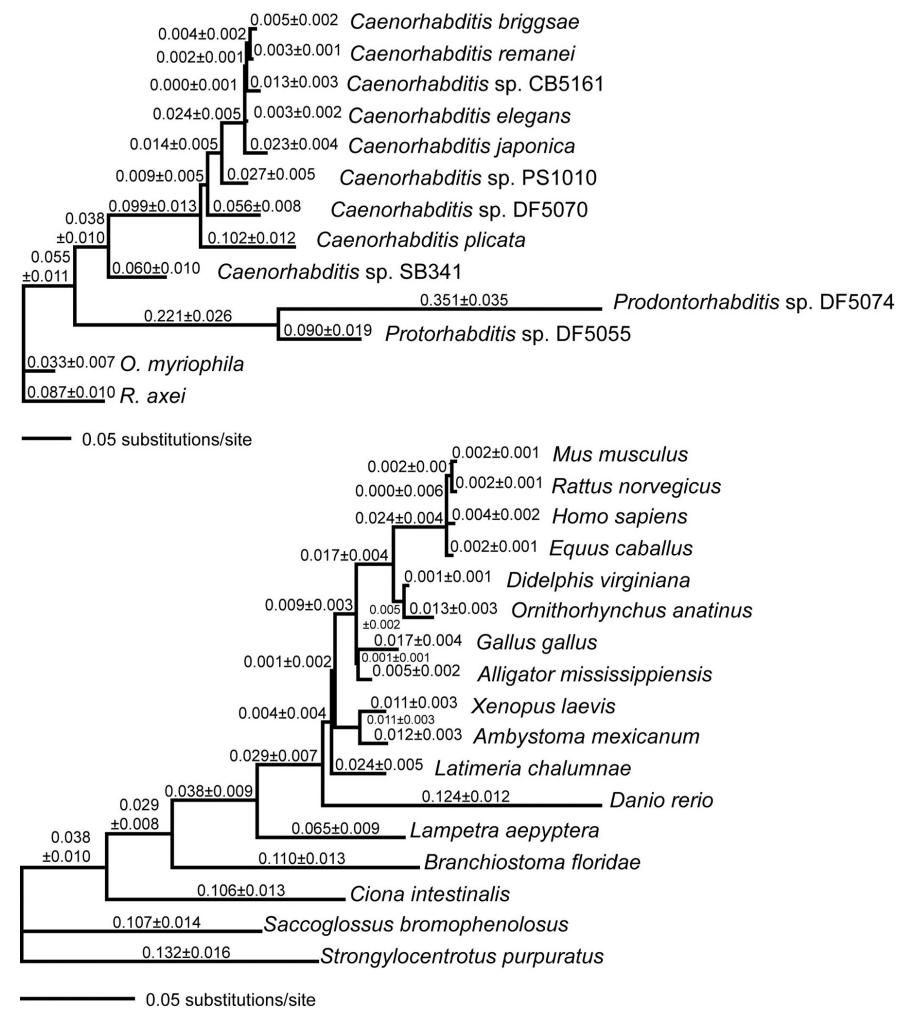

Figure 6. (A) Comparisons of evolutionary divergences in SSU rRNA genes between species pairs within nematodes (red) and deuterostomes (green). Pairwise divergences were estimated by summing lengths of intervening branches between taxa in (B); standard deviations (error bars) were calculated as the square root of the sum of the squares of the SDs of these branches. (B) Likelihood phylograms for SSU rRNA genes used to calculate the divergences graphed in (A). Species relationships within each group were assumed. A general time-reversible model (accounting for rate heterogeneity across sites with some fraction of invariant sites, $G T R+I+\Gamma$ ) was used to estimate branch lengths (substitutions per site \pm 1 standard deviation). Divergences between any pair of taxa were then calculated as the sum of the branch lengths separating those taxa on the tree. (Modified from Kiontke, 2004). Right-click or control-click to see larger image. 


\section{Resources for comparative biology using rhabditids}

Arguably the most important tools for comparative studies using emerging model systems are the organisms themselves. Genome sequences, interactome maps, cDNA libraries, and other such molecular data and resources are important tools for functional genomics. Ultimately, however, in vivo understanding of gene and genome function particularly with respect to mechanisms and patterns of gene expression, development and behavior - requires analysis in the context of the living system itself. Powerful comparative approaches depend on the availability of multiple, phylogenetically well-defined, living species with clearly defined, standardized strains that can be archived and shared. The Caenorhabditis Genetics Center (CGC) provides many stocks of wild strains that can be easily maintained cryogenically. We are also developing a stock collection of rhabditid species, the New York University Rhabditidae Collection (NYURC) that is as thoroughly representative of rhabditid phylogeny as possible. The NYURC curates strains that are difficult to culture or archive cryogenically along with data about ecology, biology, morphology, etc., for all rhabditids in culture.

\section{Acknowledgments}

We thank the National Science Foundation and the Human Frontier Science Program for supporting this work. Although many people have been important to this work, we especially thank Marie-Anne Félix, W. Kelley Thomas and Walter Sudhaus for their important collaborations and contributions. We thank Byron Adams and Steve Nadler for providing excellent suggestions in their reviews of the manuscript.

\section{References}

Andrássy, I. (1983). A Taxonomic Review of the Suborder Rhabditina (Nematoda: Secernentia) (Paris: ORSTOM).

Baldwin, J.G., Frisse, L.M., Vida, J.T., Eddleman, C.D., and Thomas, W.K. (1997). An evolutionary framework for the study of developmental evolution in a set of nematodes related to Caenorhabditis elegans. Mol. Phylogenet. Evol. 8, 249-259. Abstract Article

Blaxter, M.L., De Ley, P., Garey, J.R., Liu, L.X., Scheldeman, P., Vierstraete, A., Vanfleteren, J.R., Mackey, L.Y., Dorris, M., Frisse, L.M., et al. (1998). A molecular evolutionary framework for the phylum Nematoda. Nature 392, 71-75. Abstract Article

Cho, S., Jin, S.W., Cohen, A., and Ellis, R.E. 2004. A phylogeny of Caenorhabditis reveals frequent loss of introns during nematode evolution. Genome Res. 14, 1207-1220. Abstract Article

Coghlan, A., and Wolfe, K.H. (2004). Origins of recently gained introns in Caenorhabditis. Proc. Natl. Acad. Sci. USA 101, 11362-11367. Abstract Article

De Ley, P., and Blaxter, M. (2002). Systematic position and phylogeny. In The Biology of Nematodes, D.L. Lee, ed. (New York: Taylor \& Francis), pp. 1-30.

Evans, D., Zorio, D., MacMorris, M., Winter, C.E., Lea, K., and Blumenthal, T. (1997). Operons and SL2 trans-splicing exist in nematodes outside the genus Caenorhabditis. Proc. Natl. Acad. Sci. USA 94, 9751-9756. Abstract Article

Fitch, D.H.A., Bugaj-Gaweda, B., and Emmons, S.W. (1995). 18S ribosomal RNA gene phylogeny for some Rhabditidae related to Caenorhabditis. Mol. Biol. Evol. 12, 346-358. Abstract

Fitch, D.H.A. (1997). Evolution of male tail development in rhabditid nematodes related to Caenorhabditis elegans. Syst. Biol. 46, 145-179. Abstract

Fitch, D.H.A. (2000). Evolution of "Rhabditidae" and the male tail. J. Nematol. 32, 235-244.

Fitch, D.H.A., and Thomas, W.K. (1997). Evolution. In C. elegans II, D. L. Riddle, T. Blumenthal, B.J. Meyer and J.R. Priess, eds. (Cold Spring Harbor, N.Y.: Cold Spring Harbor Laboratory Press), pp. 815-850.

Fürst von Lieven, A. (2002). The sister group of the Diplogastrina (Nematoda). Russ. J. Nematol. 10, $127-137$. 
Jones, C.C., Rosen, T., and Greenberg, C. (1991). Cutaneous larva migrans due to Pelodera strongyloides. Cutis 48, 123-126. Abstract

Kiontke, K., Gavin, N.P., Raynes, Y., Roehrig, C., Piano, F., and Fitch, D.H.A. (2004). Caenorhabditis phylogeny predicts convergence of hermaphroditism and extensive intron loss. Proc. Natl. Acad. Sci. USA 101, 9003-9008. Abstract Article

Nayak, S., Goree, J., and Schedl, T. (2005). fog-2 and the evolution of self-fertile hermaphroditism in Caenorhabditis. PLoS Biol. 3, e6. Abstract Article

Nguyen, C.Q., Hall, D.H., Yang, Y., and Fitch, D.H.A. (1999). Morphogenesis of the Caenorhabditis elegans male tail tip. Dev. Biol. 207, 86-106. Abstract Article

Rogozin, I.B., Wolf, Y.I., Sorokin, A.V., Mirkin, B.G., and Koonin, E.V. (2003). Remarkable interkingdom conservation of intron positions and massive, lineage-specific intron loss and gain in eukaryotic evolution. Curr. Biol. 13, 1512-1517. Abstract Article

Sommer, R.J. (2000). Comparative genetics: a third model nematode species. Curr. Biol. 10, R879-R881. Abstract Article

Stein, L.D., Bao, Z., Blasiar, D., Blumenthal, T., Brent, M.R., Chen, N., Chinwalla, A., Clarke, L., Clee, C., Coghlan, A., et al. (2003). The genome sequence of Caenorhabditis briggsae: a platform for comparative genomics. PLoS Biol. 1, e45. Abstract Article

Sudhaus, W., and Fitch, D. (2001). Comparative studies on the phylogeny and systematics of the Rhabditidae (Nematoda). J. Nematol. 33, 1-70.

Sudhaus, W., and Hooper, D.J. (1994). Rhabditis (Oscheius) guentheri sp. n., an unusual species with reduced posterior ovary, with observations on the Dolichura and Insectivora groups (Nematoda: Rhabditidae). Nematologica 40, 508-533.

Sudhaus, W., and Kiontke, K. (1996). Phylogeny of Rhabditis subgenus Caenorhabditis (Rhabditidae, Nematoda). J. Zoo. Syst. Evol. Research 34, 217-233.

Sudhaus, W., and Schulte, F. (1988). Rhabditis (Pelodera) strongyloides (Nematoda) als Verursacher von Dermatitis, mit systematischen und biologischen Bemerkungen über verwandte Arten. Zool. Jahrb. (Syst.) 115, 187-205.

All WormBook content, except where otherwise noted, is licensed under a Creative Commons Attribution License. 